\title{
FOXP1 and ARID3A Collaborate to Activate Transcription of Core Embryonic Stem Cell Factors in Activated B-Cell Diffuse Large B cell Lymphoma
}

\section{Deng W, Dekker JD, Rhee C and Tucker HO* \\ Department of Molecular Biosciences, The University of Texas at Austin, USA}

*Corresponding author: Haley 0 Tucker, Department of Molecular Biosciences, the University of Texas at Austin, 1 University Station A5000, Austin TX 78712, USA, Tel: 512-475-7706; Fax: 512-475-7707; Email: haleytucker@austin.utexas.edu

\section{Research Article}

Volume 2 Issue 2

Received Date: July 06, 2018

Published Date: July 23, 2018

DOI: $10.23880 /$ jes- 16000110

\section{Abstract}

Embryonic development requires establishment of networks of stem cells via coordination of "Core" transcription factors (OCT4, SOX2, KLF4, NANOG and c-MYC; abbreviated as OSKNM) with a complex array of auxiliary and epigenetic modulators. We showed previously that down regulation of the ARID3A transcription factor is critical for embryonic stem cell (ESC) growth and differentiation to trophectoderm via direct transcriptional repression of OSKNM. FOXP1 and ARID3Aare two differentially expressed genes that define the most aggressive, Activated B Cell subset of Diffuse Large B Cell Lymphoma (ABC-DLBCL). FOXP1, a well-documented "gold standard of the ABC-subtype, more recently was identified as a regulator of pluripotency, whereas ARID3A function in ES biology is well documented. We demonstrate here that ARID3A is a directly activated target ofFOXP1. Reduction of ARID3Aexpression in ABC-DLBCL tumors, but not in the more benign, Germinal Center (GCB-DLBCL) subset, leads to loss of proliferation and down regulation of several OSKNM factors. Our results suggest that modulation of ARID3A levels may provide both prognostic and therapeutic options for a malignancy representing the most prevalent and aggressive non-Hodgkin's lymphoma worldwide.

Keywords: B-Cell; Embryonic Stem Cell; Pluripotency

\section{Introduction}

Reprogramming of somatic cells to ESC-like induced Pluripotent Stem Cells (iPSCs) now can be generated routinely from tissue biopsies or tumor samples [1]. Examples include Familial Dysautonomia [2] Fragile X syndrome [3] Down's syndrome [4,5] and several neurological diseases [5,6]. Other than cardiovascular diseases, cancer is the leading cause of death in the USA [7]. Unfortunately, most therapies developed in mouse models have failed in clinical trials, emphasizing the need for new therapeutic models and biomarkers [8]. 


\section{Journal of Embryology \& Stem Cell Research}

Diffuse Large B Cell Lymphoma (DLBCL), is the most common aggressive non-Hodgkin's lymphoma, comprising $\sim 37 \%$ of all Non-Hodgkin's Lymphomas [9]. DLBCL is categorized into distinct subtypes based on similarities with normal B cell expression and signatures of critical transcription factors (TFs) [10-14]. The two major categories, Germinal Center B Cell (GCB) and Activated B-Cell (ABC) DLBCL, have distinct pathogeneses. ABC-DLBCL is the more aggressive subtype, as current multi-agent chemotherapy achieves $\sim 80 \% 3$-y survival for GCB-DLBCL but only $45 \%$ for ABCDLBCL [15], with most cases relapsing with refractory disease $[11,15,16]$.

There has been a gradual expansion of the number of "gold standard" classifiersfor ABC-DLBCL [5,16-26]. One such factor, which we initially characterized in the context of cardiovascular transcriptional regulation [27,28], is FOXP1. While receiving considerable attention as a transactivator of critical ABC-subtype identifier genes [9,11,16,29-33], FOXP1 recently has been implicated in ESC regulation. In both human and mouse, Gabut, et al. [34] observed that an alternatively spliced isoform of FOXP1, expressed specifically in ESCs promotes expression of several essential pluripotency TFs as well as stimulates somatic cell reprogramming to iPSCs. Recent reports documenting its regulation of hematopoietic stem cell expansion [35] and embryonic neural stem cell differentiation [36] indicate that FOXP1 has the capacity to alter the transcription networks of uncommitted cells to sustain their expansion and transition between proliferation and differentiation.

We originally identified AT-Rich Interactive Domain 3A (ARID3A) as a TF that regulates immunoglobulin heavy chain transcription and hematopoietic stem cell development [37-40]. We later observed that loss of ARID3A led to reprogramming of fibroblasts via derepression of OSKNM factors [41]. ARID3A loss also enhanced standard OSKNM reprogramming of MEFs toiPSCs [42] both results suggesting that ARID3A is a repressor of reprogramming [43] ARID3A is required for the maintenance of self-renewal and differentiation of ES cells totrophoblast stem cells in both mouse and human via direct transcriptional down regulation of OSKNM [44,45]. ARID3A-mediated trans-differentiation/direct reprogramming of ESC sacts via a two-step mechanism: Repression of ESC-specific genes through decommissioning of active enhancers in ESCs, followed by activation of ESC-specific genes through Pioneer Factor activity [46].

An increasing number of studies have implicated OSKNM factors in various malignancies [47-52], but only a single study has been reported in DLBCL. In that analysis, Williams and colleagues [53] observed OCT4 expression in 2/11 (18\%) testicular DLBCLs and in 6/134 (4.5\%) nontesticular DLBCLs with a tendency toward ABC-DLBCL classification.

We show here that the "gold standard" ABC-DLBCL TF FOXP1 represses transcription of ARID3A, which in turn, results in proliferative loss of ABC-DLBCL tumors as well as in up regulation of several Core pluripotency factors. We discuss these unanticipated findings in the context of the cancer stem cell model with attention to potential therapeutic extensions.

\section{Results and Discussion}

\section{FOXP1 directly transactivatesArid3aand $c$-Myc, and indirectly up regulates 0 ct4}

We compared transcripts directly activated or repressed by FOXP1following its shRNA-mediated knockdown (KD) in an extended panel of 12 ABC- and 16 GCB-DLBCL tumor lines previously reported in Dekker, et al. [33] and detailed in Methods and Materials Figure 1A. Clustering based on correlation of expression patterns allowed us to segregate ABC- and GCB-DLBCL types with high concordance. FOXP1, ARID3A and OCT4 were among the 45 down-regulated transcripts at least 2-fold in ABCDLBCL lines but not among the 115 genes activated by FOXP1 KD in GCB-DLBCL lines Figure 1B and C; red and green, respectively).

ChIP-Seq was performed as previously described [33] (detailed in Materials and Methods) employing antiFOXP1 rabbit polyclonal antibody [27] for pull downs. We observed strong peaks within Arid3a and c-myc promoter regions in ABC- but not in GCB-DLBCL chromatin Figure 1D, S-Figure 1. No peaks were observed for Oct4 in either DLBCL subtype Figure 1D. None of the remaining ABCphenotype of Figure $1 \mathrm{~B}$ has been established as stem cell candidates, with the exception of OCT2. OCT2 was identified in an ESC over expression screen as a TF that repressed ESC differentiation to induce neuronal differentiation [54]. OCT2 is directly repressed by FOXP1 S-Figure 2 but was not investigated further in this study. 


\section{Journal of Embryology \& Stem Cell Research}

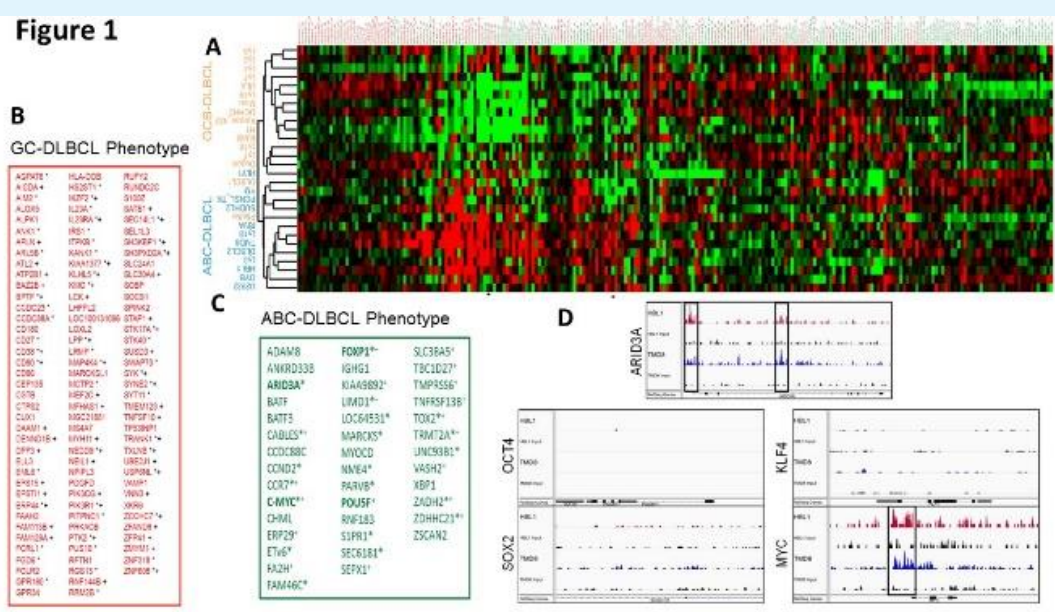

Figure 1: FOXP1 directly transactivates Arid3a and c-Myc, and indirectly up regulates Oct4.

A. Expression of FOXP1 is sufficient to differentiate ABC- from GCB-DLBCL. FOXP1 shRNA knockdown (KD) provided unbiased clustering of endogenous target expression levels in a panel of 12 (green) ABC- and 16 GCB-DLBCL (red) lines: B, C. FOXP1, ARID3A, OCT4 and c-MYC (in bold) are among 45 transcripts down-regulated in ABC-DLBCL lines (green), but not in GCB-ABC lines (red); +, down regulated at least 2-fold; *, directly down-regulated as determined by ChIP seq; D. FOXP1 is recruited to regulatory loci of Arid3a and $c$-Myc, but not that of Oct4. ChIP-seq peaks from the TMD8 and HB-1 ABC-DLBCL lines are shown as read density tracks. Aligned, significant peaks are boxed.

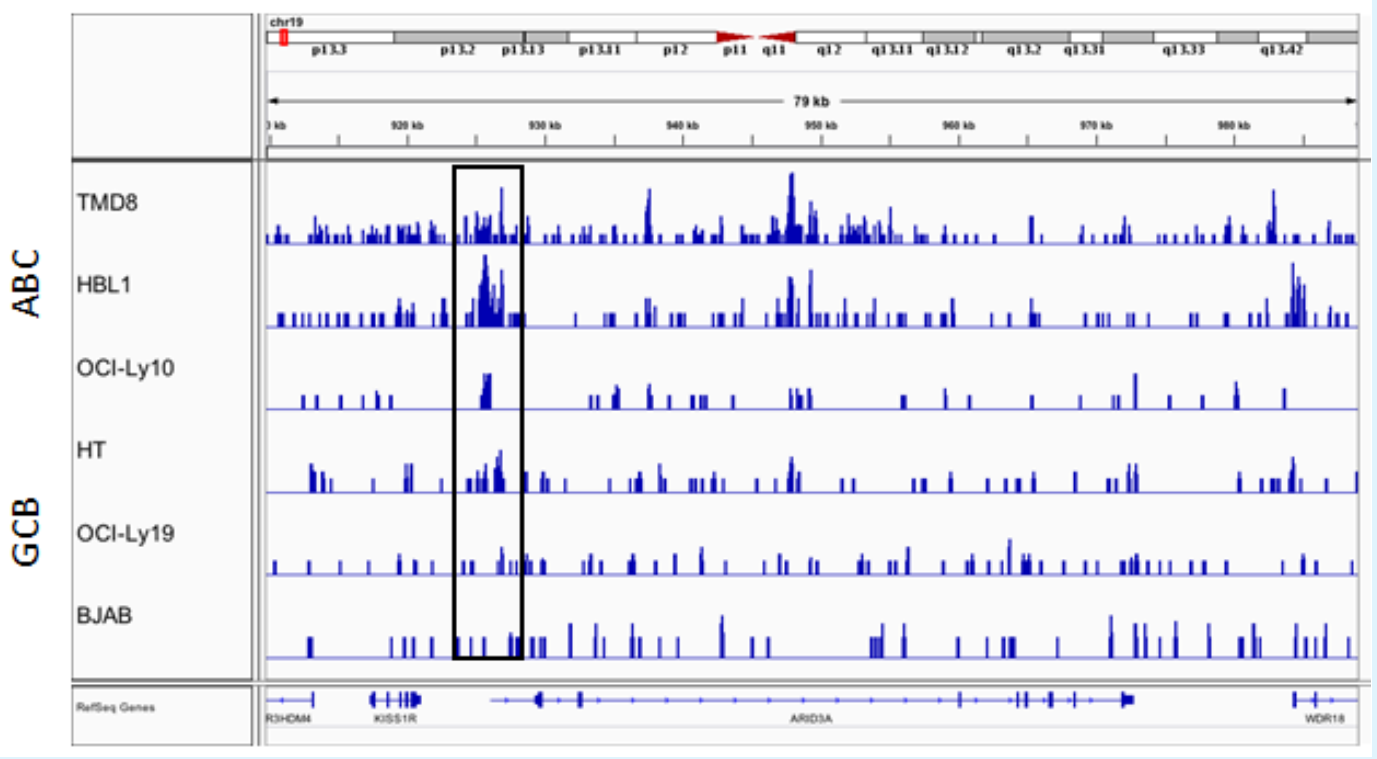

S-Figure 1: ARID3A is a direct transcriptional target of FOXP1 in ABC- but not GCB-DLBCL. ChIP-seq was carried out as described in Figure 1, Materials and Methods and previously33. A promoter-localized Arid3a binding peak (boxed) is observed in ABC- lines TMD8, HB1, OCI-Ly10, but is weak or absent in GCB-DLBCL lines HT, OCI-Ly19, and BJAB. 


\section{Journal of Embryology \& Stem Cell Research}

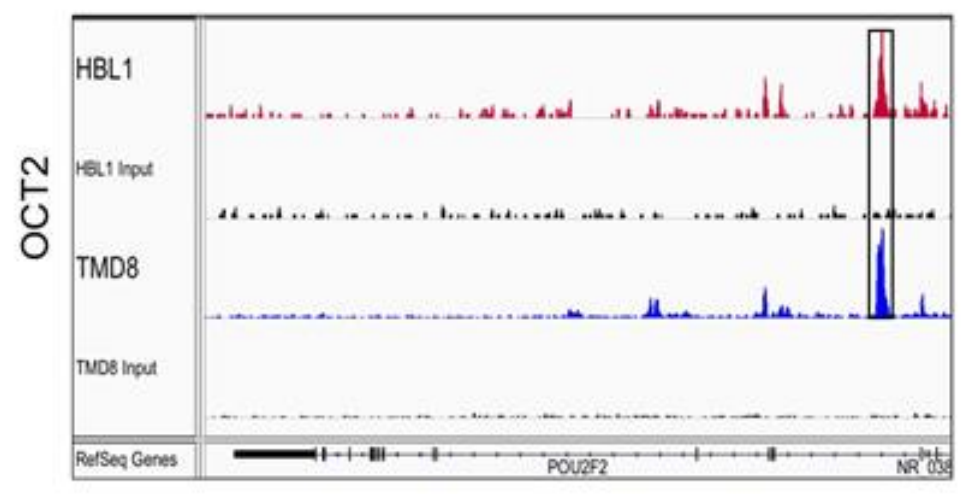

S-Figure 2: Oct2 Is A Direct Transcriptional Target Of FOXP1.

Chip-Seq peaks from TMD8 and ABC-DLBCL Lines are shown as Read Density Tracks. Aligned, Significant Peaks are boxed

These data indicate that ABC- but not GCB-DLBCL tumors express detectable levels of ARID3A and c-MYC transcripts under direct transcriptional control of FOXP1. OCT4 appears to be up regulated indirectly, perhaps by FOXP1 induction of message stabilization-a phenomenon that has been observed previously for OCT4 [55]. Indeed, a variety of additional processes have been identified that control and alter post-transcribed core factors, including phosporylation and mature protein degradation by proteasomes [55-57]. The role of post- transcriptional regulators and protein networks in the maintenance of pluripotency is still largely unknown, especially in human cells.

\section{ARID3A loss is lethal to ABC- but not to GCB- DLBCL cells}

As the next step in investigating ARID3A in the above context, we determined the effect of its loss on DLBCL viability.

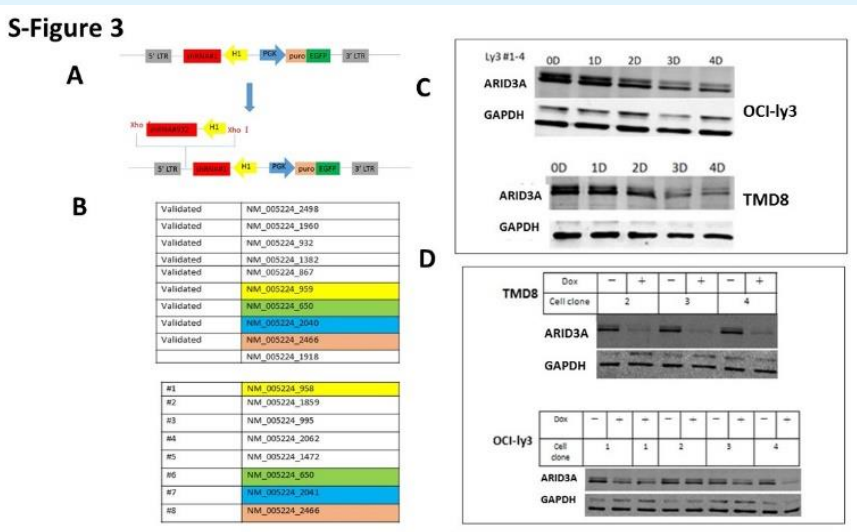

S-Figure 3: Optimization of ARID3A knockdown vectors and kinetics. A. Construction strategy. Mao of retroviral shRNA vector Prsmx [58] indicated where the shRNAs of A. was cloned; B. Commercial shRNA sequences tested for ARID3A KD efficiency after cloning into pRSMX. NM-005224 is the mouse gene bank accessibility number; numbers at the end are the targeted amino acids of ARID3A. Colors denote sequences cloned into pRSMX and tested for KD efficacy; C. Western blots analysis of the time course of successful KD. The indicated clones were generated by limiting dilution, and then these RNAs were transduced into pRSMX for 4 days prior to testing; GAPDH served as control; D. Western blot analysis to determine KD efficiencies of ARID3A shRNA clones following retroviral transduction into ABC-DLBCL lines, OCI-Ly3 and TMD8. Cellular clones 2-4 provided robust KD and were used for subsequent experiments. GAPDH served as a loading control 


\section{Journal of Embryology \& Stem Cell Research}

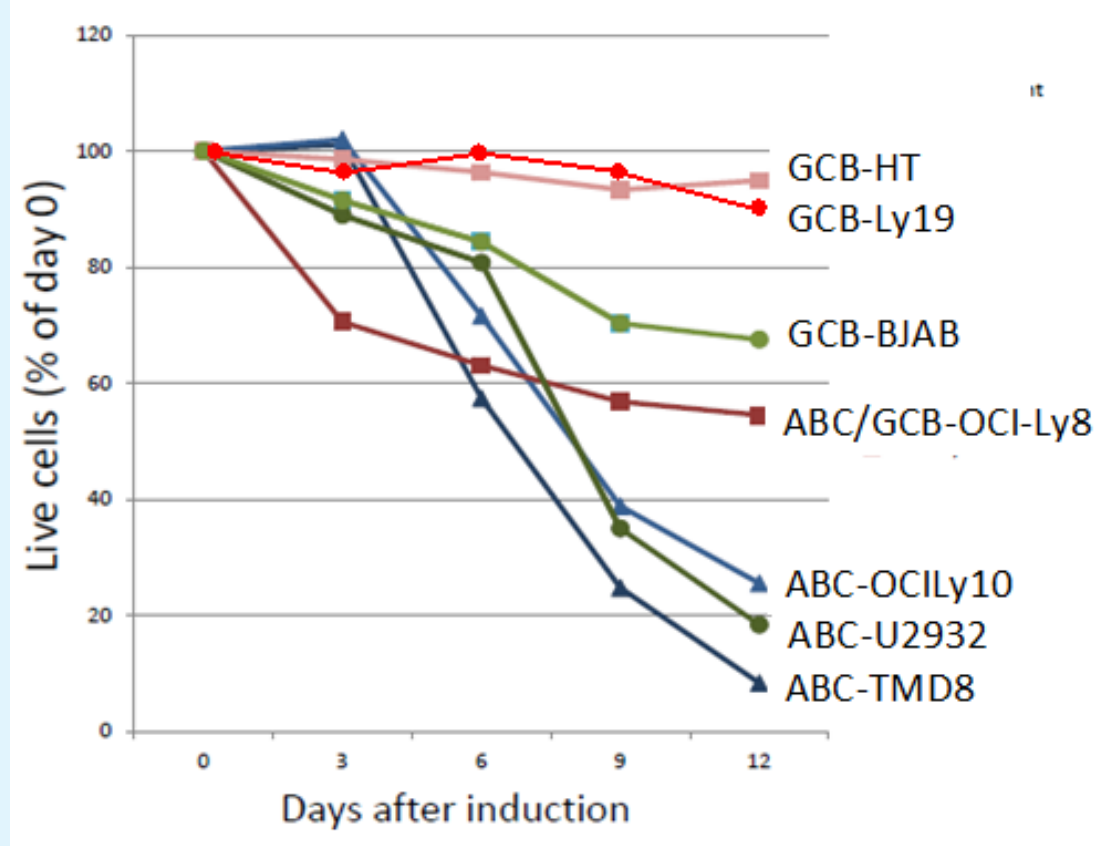

Figure 2: E. Loss of cell viability of ABC but not GCB-DLBCL lines following 12 days of inducible FOXP1 KD. Percent cell viabilities were assessed by flow cytometry of constitutive GFP levels independently encoded by the FOXP1 shRNA vector pRSMX.

We constructed several ARID3A dox-inducible, retroviral ARID3 KD vectors S-Figure 3. Following their optimization in ABC- and GCB-DLBCL lines S-Figure 3, infected ABC- and GCB-DLBCL lines were measured by flow cytometry of the GFP-tagged retroviruses for viability. As shown in Figure 2, when measured over a time course commensurate with induction kinetics $S$ Figure 3, expression of sh-ARID3A led to significant death of ABC-DLBCL cells but only modest loss of GCB-DLBCL cells. Death of the ABC/GCB intermediate cell line, OCILy8, was in between the two subtypes (Figure 2).

The data indicate that consistent with its expression pattern Figure1, ARID3A is essential for proliferation of ABC- but not for GCB-DLBCL cells. Similar, DLBCL subtype-specific defects in proliferation have been observed previously. For example, RNAi-mediated KD of IKK $\beta$ expression was toxic to ABC-, but not to GCB-DLBCL cell lines [59]. Further, the CARD11-BCL10-MALT1 (CBM) complex an established upstream activator of IKK activation and proliferation-was toxic exclusively to ABCDLBCL tumors [60].

\section{ARID3A Loss Alters Transcription of Core ESC pluripotency in ABC- but not in GCB-DLBCL}

Our previous studies showed that ARID3A is essential for the first cell fate decision, in that it's over expression alone is sufficient to drive trans-differentiation of ESC to trophectoderm (TE) stem cells [44,45]. Conversely, shRNA-mediated ARID3A KD led to derepression of OSKNMTFs while repressing transcription of TE-specific factors $[44,45]$. As the next step in addressing the consequence of ARID3A loss in DLBCL, we coupled our inducible ARID3A KD system described above with RNAsequencing to analyze two well-established ABC-DLBCL lines, TMD8 and OCI-Ly3.

Reduction in ARID3A RNA expression resulted in significant alteration of a number of gene families, including some observed in previous studies [38-40,45], including inflammatory mediators, chemokines, cytokines and adaptive immune response mediators S-Figure 4A. However, these analyses detected no alteration of OSKNM or other pluripotency regulators such as observed in the FOXP1 KD of Figure 1. 


\section{Journal of Embryology \& Stem Cell Research}

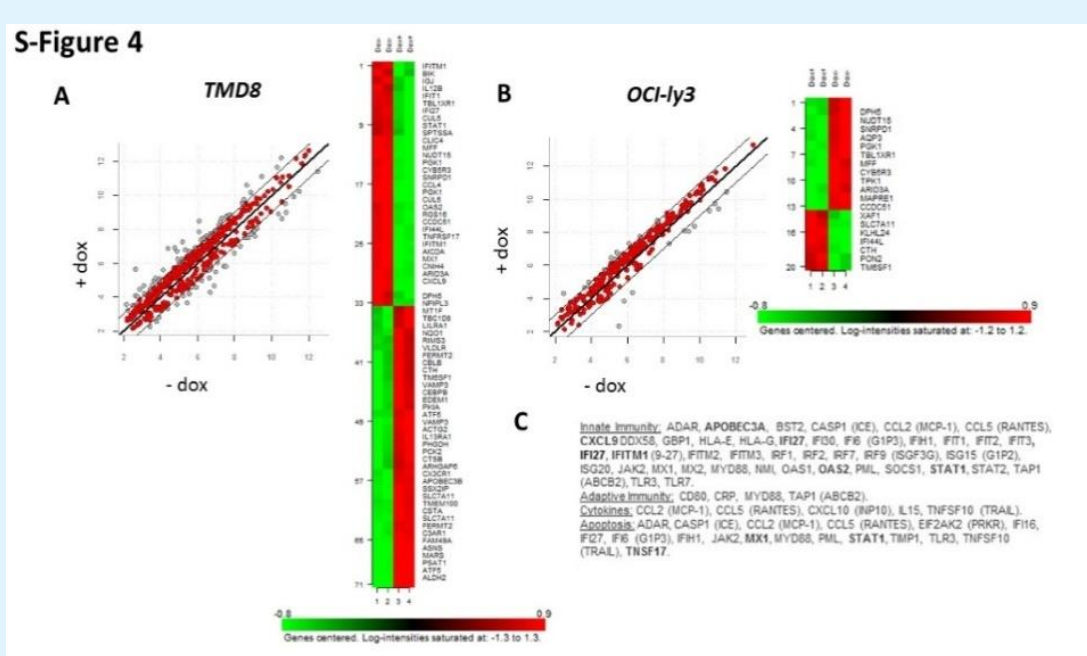

S-Figure 4: Microarray Analysis of Inducible Shrna Knockdown of ABC-DLBCL Lines.

A, B. Targets obtained follow ARID3A KD of ABC-DLBCL lines TMD (left) and OCI-Ly3 (right). Target frequency distributions shown were generated after $(+)$ or prior to (-) Doxycycline (Dox) administration for 4days to mediate ARID3A KD. Off diagonal gray dots indicate target transcripts which were modulated at a minimum of two-fold. Shown to the right are representative heat maps for each ABC-DLBCL analyses; C. Summary of significant ARID3A targets modulated after 4 days of Dox treatment and arranged into functional groups. Most highly modulated transcripts are underlined.

These Results Suggested Either That: 1) ARID3A was Not an Intermediate in The Potential FOXP1-OSKNM Pathway; 2) That ARID3A Manifests its Effects At The Post transcription Level and Such Modifications are Not Detectable By Microarray; 3) That OSKNM Expression Levels In ABC-DLBCL Were Too Modest To Detect.

Indeed, the latter hypotheses appear to be correct. We coupled the same inducible ARID3A KD system with highly sensitive Q-RTPCR analyses which were optimized in ESC for OSKNM factor detection. As shown in (Figure 3 ), after 4 days of doxycycline (dox) induction, ARID3A KD led to significant reduction of OCT4, SOX2, NANOG and KLF4 in the ABC-DLBCL tumor, HB-1. The reductions observed were even more robust that the KD level of ARID3A itself. In ABC- TMB-8 cells, in which ARID3A KD was equivalent to that in HB- 1 , we observed modest but reproducible loss of OCT4 and SOX2, significant loss of NANOG, and modest down regulation of KLF4. In the ABCline U2932, where we achieved the most robust ARID3A $\mathrm{KD}$, each factor was reproducibly reduced with particularly significant reduction achieved for KLF4.In contrast, Q-PCR of three GCB-DLBCL transduced lines (BJAB, HT and Ly19) detected no or only modest expression of any of the OSKNM factors tested.

We conclude that $A B C$-DLBCL tumors express variable levels of core pluripotency factors, whereas GCB-DLBCL lines do not. The results are in agreement with the data of (Figure 1) with one exception: We observed no variation in ESC of ARID3A-modulated c-MYC expression (data not shown). In this regard, several groups have shown that the overall ESC gene expression signature is comprised of smaller sub-signatures represented as $\sim 100-200$ gene "modules" [59-62]. In addition to core factors (CORE), Kim, et al. [62] divided ESCs according toexpression of cMYC-related factors, as they found that the c-MYC module is largely independent of the core pluripotency network-a result consistent with our failure to detect c-MYC alteration in Figure 3 Further, c-MYC was the most active signature shared by ESCs and Cancer Stem Cells [62-65]. 


\section{Journal of Embryology \& Stem Cell Research}

Figure 3

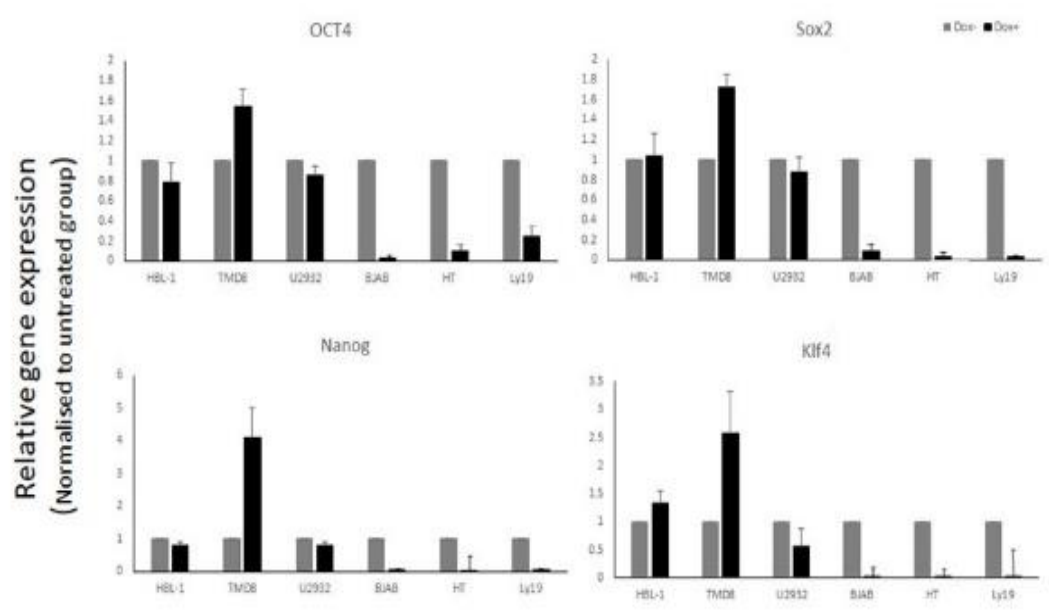

Figure 3: Loss of ARID3A Leads to Significantly Yet Variable Loss of Pluripotency Factors in ABC- But Not in GCBDLBCL.

After Optimizing Stable ARID3A Expression and Optimal KD Kinetics of ABC-DLBCL Lines (HB-1, TMD8, And OCL-Ly3; S-Figs. 2 And 3), RNA Was Isolated From Each Line At Maximal Time Points And Then Subjected To Q-RTPCR For Pluripotency Factors (OCT4, SOX2, NANOG And KLF4). ARID3A Levels Were Set As 1.0 And Levels of Pluripotency Factor Rnas Were Compared On The Y-Axis By Relative Expression Normalized To Controls (Without Dox Induction Of ARID3 Expression). Note: The Absolute Values of the Y-Axes Vary For Each Factor, Indicating Higher or Lower UnManipulated Expression Prior To KD.

\section{Relevance to Cancer Stem Cells, Prognosis and Therapy}

Cancer stem Cells (CSCs) were first identified in Leukemia [70]. Their existence as since been documented in an array of solid tumors [71-75]. CSCs share properties many properties with ESCs but differ in their ability to survive in hostile environments and to undergo metastasis. We have shown here that the ARID3A TF shares properties with both ECSCs and CSCs in its capacity to direct critical ESC-requiring gene expression in a neoplastic environment.

Considerable data has accumulated that consolidates the functions of core pluripotency transcription factors as adverse prognostic factors in cancer. For example in colorectal cancer, the mRNA-binding proteins LIN28 and Musashi, as well as the deacetylase epigenetic modifier SIRT1, which are known to be associated with stem cell proliferation, recently have been implicated as prognostic in colorectal cancer [72-74]. OCT4 has been shown to be a predictive biomarker for bad prognosis in colorectal cancer, hepatocellular carcinoma, esophageal squamous cell carcinoma and ovarian cancer [75-78]. SOX2 and
NANOG are expressed in and appear to play critical roles in lung, breast, and endometrial tumorigenesis [79-82].

Although ESC core factors are expressed in cancer and correlate with functions ascribed to neoplastic states, do these factors actually play a crucial mechanistic role in cancer initiation and sustenance? Clearly an improved understanding of the features shared by FOXP1, ARID3A and conventional core factors with neoplastic cells is of significant clinical interest. The factors themselves, or common pathways shared in pluripotency by malignant cells, might serve as future targets for anti-cancer drugs. Such goals will require further understanding of shared ESC and CSC signaling pathways, whether activities of core signatures might be shifted via chemical or genetic modifiers, and most importantly, their efficacy in developing new cancer therapeutics.

\section{Methods and Materials}

\section{RNA Isolation}

Total RNA was extracted from DLBCL tumors using TRIzol reagent (Invitrogen). Oligo-dT-primed cDNA was 


\section{Journal of Embryology \& Stem Cell Research}

prepared using Super Script III First-Strand Synthesis System for RT-PCR (Invitrogen). Taq polymerase (New England Biolabs) and a Perkin-Elmer 2700 thermo cycler were used to amplify transcripts.

\section{shRNA knockdowns}

Cells were stably transduced with retroviruses expressing the bacterial tetracycline repressor $\left(\mathrm{TET}^{\mathrm{R}}\right)$ and the blasticidin resistance gene, and then retrovirally transduced with Phoenix-E packaged pRSMX-PG TETRinducible vectors [58]. The vectors encoded shRNAs listed in S-Figure 3 targeted to FOXP1, ARID3A or scrambled shRNA controls (termed dox controls). Dox (1-10 $\mu \mathrm{g} / \mathrm{ml})$ was applied for induction of shRNA expression in each experiment. Cells were harvested for RNA isolation at multiple time points. Knockdowns (KD) were considered sufficient for analysis when each reached $50 \%$ or lower relative expressions compared to controls, as judged by RT-qPCR. SYBR green real time Q-PCR was performed after high capacity cDNA reverse transcription according to manufacturer's protocols (Applied Biosystems Cat. \#'s 4385618 and 4368814). Sequences of the 21bp DNA stems of the shRNAs used for FOXP1 KD were: \#26 GGCGGTCCAACGGAGAGACGC, \#36 GACCTCGCCCACGCCCAGCAG, and \#42 GCAGCAACAGTGGCATCTCAT, or an ineffective nonspecific control shRNA. FOXP1 primers were: F: 5'AAAACATGCAGAGCAGCCAC-3' and R: 5' TGGCTAAGTTGCCCAGAGTG-3'. ARID3A primers were F: 5'-TGAGTTCCCAAGGTCTGTGTGTTC-3' and R: 5'GGATCTCGTACCGTAAATGGCAGT-3'

Western blots. Western blots were performed by common procedures; FOXP1 band densities were quantified with ImageJ [83]. Antibodies used were antiFOXP1 mAb 1B3 [27] and an affinity purified anti-ARID polyclonal [39].

\section{Microarray}

Following purification as described above, total RNA was subjected to clean-up (RNA easy; Qiagen). Labeled complementary RNA was prepared as described in the Agilent $4 \times 44$ microarrays manual. Gene expression was determined by comparing signal passing confidence criteria (provided by the manufacturer) from dox-induced cells, with an ineffective control shRNA (Cy3), to doxinduced cells bearing FOXP1 or ARID3A targeted shRNAs (Cy5) at each time point. Data were analyzed as $\log 2$ ratio (Cy5/Cy3). Genes with $\log 2$ values of \pm 0.3 change in at least 5/12 microarray $\mathrm{KD}$ samples/cell line were considered modulated. Hierarchical clustering of enriched gene subsets by Pearson correlation allowed segregation of ABC- and GCB-DLBCL primary isolates to varying degrees. Genes that were both directly bound and downregulated upon FOXP1 loss in TMD8 and HBL1 clustered 21 of $32 \mathrm{ABC}$ primary isolates as well as a majority of GCB primary isolates, as indicated by $\mathrm{ABC}$ (red) and GCB (blue) designation above the heatmaps in Figure 1.

\section{ChIP and RNA-sequencing}

ChIP pull downs were performed with an anti-FOXP1 rabbit polyclonal antibody as previously described [33]. RNA-seq was carried out as previously described [33]. Briefly, cDNA was prepared using the Ultra-Low kit from Clontech (Mountain View, CA). Library preparation employed the Nextera DNA Library Preparation kit from Illumina (San Diego, CA). cDNA was analyzed by deep sequencing using Illumina sequencing technology. Data were analyzed using a high-throughput next-generation sequencing analysis pipeline: FASTQ files were aligned to the mouse genome (mm9, NCBI Build 37) using Top Hat [84]. Gene expression profiles for the individual samples were calculated with Cufflinks [85] as RPKM values. Two subtypes, $\mathrm{ABC}$ or GCB were determined based on a previous study [33]. Expression data were split by subtypes. For each subtype, Gene Set Enrichment Analysis (GSEA) [86] was used to rank genes by correlation with FOXP1 expression and to evaluate the enrichment of the pre-defined gene sets.

\section{Q-RT-PCR}

Human OSKMN factors were amplified by real-time PCR performed with Platinum SYBR Green qPCR Super Mix-UDG with ROX (Invitrogen) according to manufacturer's instructions. An ABI7300 Real-Time PCR System (Applied Biosystems) was employed for signal detection. Human OSNKM primer sequences $[87,88]$ are: OCT4 F: 5'-TCT TTC CAC CAG GCC CCC GGC TC-3'; R: 5'TGC GGG CGG ACA TGG GGA GAT CC

SOX2 F: 5'-TAG AGC TAG ACT CCG GGC GAT GA-3'; R: 5'TTG CCT TAA ACA AGA CCA CGA AA-3'

NANOG F: 5'-AGG GTC TGC TAC TGA GAT GCT CTG-3'; R: 5'-CAA CCA CTG GTT TTT CTG CCA CCG-3'

KLF4F: 5'-CAC CAT GGA CCC GGG CGT GGC TGC CAG AAA3'; R: 5'-TA GGC TGT TCT TTT CCG GGG CCA CGA-3'

C-MYC F:5'-TGA CCT AAC TCG AGG AGG AGC TGG AAT C3'; R: 5'-AAG TTT GAG GCA GTT AAA ATT ATG GCT GAA GC-31

\section{Accession Numbers}

ChIP-seq accession: GSE63257; Microarray accession: GSE64586 


\section{Journal of Embryology \& Stem Cell Research}

\section{Author Contributions}

W.D., J.D.D. and H.O.T. designed research; W.D. and J.D.D. performed research; W.D. and J.D.D. contributed new reagents/analytic tools; W.D., J.D.D. and H.O.T. Analyzed data; W.D., J.D.D. and H.O.T. Wrote the paper.

The authors declare no conflict of interest.

\section{Acknowledgements}

We thank June V. Harriss for expert assistance in the maintenance and generation of FOXP1 conditional knockout mice, and Chhaya Das and Maya Ghosh for help in ChIP experiments and cell culture. Library preparation and Illumina ChIP- and RNA-seq were performed at the NGS core of the MD Anderson Cancer Center. The work was supported by a Lymphoma Research Foundation Fellowship (300463) to J.D.D.; NIH grant R01CA31534, Cancer Prevention Research Institute of Texas (CPRIT) RP120348 and RP120459; and the Marie Betzner Morrow Centennial Endowment; and CPRIT Grant RP120348 to the MD Anderson NGS core.

\section{References}

1. Raab S, Klingenstein M, Liebau S, Linta L (2014) a comparative view on human somatic cell sources for iPSC generation. Stem Cells Int 1-2.

2. Lee G, Papapetrou EP, Kim H, Chambers SM, Tomishima MJ, et al. (2009) Modelling pathogenesis and treatment of familial dysautonomia using patient-specific iPSCs. Nature 461: 402-406.

3. Li LB, Chang KH, Wang PR, Hirata RK, Papayannopoulou T, et al. (2012) Trisomy correction in Down syndrome induced pluripotent stem cells. Cell Stem Cell 11: 615-619.

4. Chen C, Jiang P, Xue H, Peterson SE, Tran HT, et al. (2014) Role of astroglia in Down's syndrome revealed by patient-derived human-induced pluripotent stem cells. Nat Commun 5: 4430.

5. Nguyen H N, Byers B, Cord B, Shcheglovitov A, Byrne J, et al. (2011) LRRK2 mutant iPSC-derived DA neurons demonstrate increased susceptibility to oxidative stress. Cell Stem Cell 8(3): 267-280.

6. Sanchez-Danes A, Richaud-Patin Y, Carballo-Carbajal I, Jiménez-Delgado S, Caig C, et al. (2012) Diseasespecific phenotypes in dopamine neurons from human iPS-based models of genetic and sporadic Parkinson's disease. EMBO Mol Med 4(5): 380-395.
7. Nichols H, Legg TJ (2017) the top 10 leading causes of death in the United States. Med News Today 2(1): 1-9.

8. Mak I W, Evaniew N, Ghert M (2014) Lost in translation: animal models and clinical trials in cancer treatment. Am J Transl Res 6(2): 114-118.

9. Leukaemia Foundation (2017) Diffuse large B-cell lymphoma (DLBCL). Leukemia Foundation Website.

10. Alizadeh AA, Eisen MB, Davis RE, Ma C, Lossos IS, et al. (2000) Distinct types of diffuse large B-cell lymphoma identified by gene expression profiling. Nature 403(6769): 503-511.

11. Wright G, Tan B, Rosenwald A, Hurt EH, Wiestner A, et al. (2003) a gene expression-based method to diagnose clinically distinct subgroups of diffuse large B cell lymphoma. Proc Natl Acad Sci USA 100(17): 9991-9996.

12. Rosenwald A, Wright G, Chan WC, Connors JM, Campo E, et al. (2002) The use of molecular profiling to predict survival after chemotherapy for diffuse largeB-cell lymphoma. N Engl J Med 346(25): 1937-1947.

13. Lenz G, Wright GW, Emre NC, Kohlhammer H, Dave SS, et al. (2008) Molecular subtypes of diffuse large Bcell lymphoma arise by distinct genetic pathways. Proc Natl Acad Sci USA 105(36): 1352013525.

14. Lenz G, Wright G, Dave SS, Xiao W, Powell J, et al. (2008) Stromal gene signatures in large-B-cell lymphomas. The New England journal of medicine 359(22): 2313-2323.

15. Lenz G, Staudt LM (2010) Aggressive lymphomas. The New England journal of medicine 362: 1417-1429.

16. Howlader (2013) SEER Cancer Statistics Review, 1975-2010. National Cancer Institute; Bethesda MD.

17. Based on November (2012) SEER data submission. Available atseer.

18. Banham AH, Connors JM, Brown PJ, Cordell JL, Ott G, et al. (2005) Expression of the FOXP1 transcription factor is strongly associated with inferior survival in patients with diffuse large B-cell lymphoma. Clin Cancer Res 11(3):1065-1072.

19. Dave SS, Fu K, Wright GW, Lam LT, Kluin P, et al. (2006) Molecular diagnosis of Burkitt's lymphoma. The New Eng J Med 354: 2431-2442. 


\section{Journal of Embryology \& Stem Cell Research}

20. Barrans SL, Fenton JEL, Banham A, Owen RG, Jack AS (2004) Strong expression of FOXP1 identifies a distinct subset of diffuse large B-cell lymphoma (DLBCL) patients with poor outcome. Blood 104(9): 2933-2935.

21. Monti S, Savage KJ, Kutok JL, Feuerhake F, Kurtin P, et al. (2005) Molecular profiling of diffuse large B-cell lymphoma identifies robust subtypes including one characterized by host inflammatory response. Blood 105(5): 1851-1861.

22. Hummel $M$, Bentink $S$, Berger $H$, Klapper $W$, Wessendorf S, et al. (2006) A biologic definition of Burkitt's lymphoma from transcriptional and genomic profiling. The New England journal of medicine 354(23): 2419 -2430.

23. Salaverria I, Philipp C, Oschlies I, Kohler CW, Kreuz M, et al. (2011) Translocations activating IRF4 identify a subtype of germinal center-derived B-cell lymphoma affecting predominantly children and young adults. Blood 118(1): 139-147.

24. Gutierrez-Garcia G, Cardesa-Salzmann T, Climent F, Gonzalez-Barca E, Mercadal S, et al. (2011) Geneexpression profiling and not immunophenotypic algorithms predicts prognosis in patients with diffuse large B-cell lymphoma treated with immunochemotherapy. Blood 117(18): 4836-4843.

25. Jima DD, Zhang J, Jacobs C, Richards KL, Dunphy $\mathrm{CH}$, et al. (2010) Deep sequencing of the small RNA transcriptome of normal and malignant human B cells identifies hundreds of novel micro RNAs. Blood 116(23): e118-127.

26. Care MA, Barrans S, Worrillow L, Jack A, Westhead DR, et al. (2013) A microarray platform-independent classification tool for cell of origin class allows comparative analysis of gene expression in diffuse large B-cell lymphoma. PLoS One 8(2): e55895.

27. Schmitz R, George WW, Huang DW, Calvin A, Johnson, et al. (2018) Genetics and Pathogenesis of Diffuse Large B-Cell Lymphoma. N Engl J Med 378: 13961407.

28. Wang B, Lin D, Li C (2003) Tucker P. Multiple domains define the expression and regulatory properties of Foxp1 forkhead transcriptional repressors. J Biol Chem 278 (27): 24259-24268.
29. Wang B, Weidenfeld J, Lu MM, Maika S, Kuziel WA, et al. (2015) Foxp1 regulates cardiac outflow tract, endocardial cushion morphogenesis and myocyte proliferation and maturation. Development 131(18): 4477-4487.

30. Brown PJ, Ashe SL, Leich E, Burek C, Barrans, S et al. (2008) potentially oncogenic B-cell activationinduced smaller isoforms of FOXP1 are highly expressed in the activated B cell-like subtype of DLBCL. Blood 111 (5):2816-2824.

31. Wlodarska I, Veyt E, De Paepe P, Vandenberghe P, Nooijen P, et al. (2005) FOXP1, a gene highly expressed in a subset of diffuse large B-cell lymphoma, is recurrently targeted by genomic aberrations. Leukemia 19(8): 1299-1305.

32. Sagaert $X$, de Paepe $P$, Libbrecht L, Vanhentenrijk V, Verhoef G, et al. (2006) Forkhead box protein P1 expression in mucosa-associated lymphoid tissue lymphomas predicts poor prognosis and transformation to diffuse large B-cell lymphoma. J Clin Oncol 24 (16): 2490-2497.

33. van Keimpema M, Grüneberg LJ, Esther JM, SchilderTol, E.C.M. Oud M, et al. (2017) The small FOXP1 isoform predominantly expressed in activated B celllike diffuse large B-cell lymphoma and full-length FOXP1 exert similar oncogenic and transcriptional activity in human B cells. Haematologica 102 (3): 573-583.

34. Dekker JD, Park D, Shaffer AL, Kohlhammer H, Deng W, et al. (2016) Subtype-specific addiction of the activated B-cell subset of diffuse large B-cell lymphoma to F0XP1. Proc Natl Acad Sci U S A 113(5): E577-E586.

35. Gabut M, Samavarchi-Tehrani P, Wang X, Slobodeniuc V, O'Hanlon D, et al. An alternative splicing switch regulates embryonic stem cell pluripotency and reprogramming. Cell 147(1): 132-146.

36. Michelet F, Hattabi M, Michelet F, Mire-Nezhad A, Benyoucef A, et al. (2017) PUMILIO/FOXP1 signaling drives expansion of hematopoietic stem/progenitor and leukemia cells. Blood 129(18): 2493-2506.

37. Braccioli L, Vervoort SJ, Adolfs Y, Heijnen CJ, Basak O, et al. (2017) FOXP1 promotes embryonic neural stem cell differentiation by repressing Jagged1 expression. Stem Cell Reports 9(5): 1530-1545. 


\section{Journal of Embryology \& Stem Cell Research}

38. Herrscher RF, Kaplan MH, Lelsz DL, Das C, Scheuermann R, et al. (1996). Immunoglobulin gene expression is regulated by Bright: A B cell specific, MAR-binding transactivator that contains a new class of DNA binding domain. Genes and Development 9: 3067-3082.

39. Webb CF, Bryant J, Popowski M, Allred A, Kim D, et al. (2011) The ARID family transcription factor Bright is required for both hematopoietic stem cell and $B$ lineage development. Mol Cell Biol 31(5): 1041-53.

40. Kim PG, Matthew CC, Rhee C, Samantha JR, June VH, et al. (2016) Interferon- $\alpha$ signaling promotes embryonic HSC maturation. Blood 128: 204-216.

41. An G, Miner CA, Nixon JC, Kincade PW, Bryant J, et al. (2010) Loss of Arid3a/ARID3a function promotes developmental plasticity. Stem Cells 28: 1560-1567.

42. Popowski M, Templeton TD, Lee BK, Rhee C, Li H, et al. (2014) Arid3a/Arid3A Acts as a Barrier to Somatic Cell Reprogramming through Direct Regulation of Oct4, Sox2, and Nanog. Stem Cell Rep 2(1): 26-35.

43. Popowski M, Tucker H (2015) Repressors of reprogramming. World J Stem Cells 7(3): 541-546.

44. Rhee C, Lee BK, Beck S, Anjum A, Cook KR, et al. (2015) Arid3a is essential to execution of the first cell fate decision via direct embryonic and extra embryonic transcriptional regulation. Genes Dev 28(20): 2219-32.

45. Rhee C, Dang C, Harris J, Brown M, Kim J, et al. ARID3A is required for mammalian placenta development. Dev Biol 422(2): 83-91.

46. Rhee C, Lee B-K, Beck S, LeBlanc L, Tucker HO, et al. (2017) Mechanisms of transcription factor-mediated direct reprogramming of mouse embryonic stem cells to trophoblast stem-like cells. Nucleic Acids Res 45(17): 10103-10114.

47. Kumano K, Arai S, Hosoi M, Taoka K, Takayama N, et al. (2012) Generation of induced pluripotent stem cells from primary chronic myelogenous leukemia patient samples. Blood 119(26): 6234-6242.

48. Utikal J, Maherali N, Kulalert W, Hochedlinger $\mathrm{K}$ (2009) Sox2 is dispensable for the reprogramming of melanocytes and melanoma cells into induced pluripotent stem cells. J. Cell. Sci 122(19): 3502-3510.
49. Miyoshi $\mathrm{N}$, Ishii $\mathrm{H}$, Ken-ichi $\mathrm{N}$, Hoshino $\mathrm{H}$, Koshi Mimori, et al. (2010) Defined factors induce reprogramming of gastrointestinal cancer cells. Proc. Natl Acad Sci USA 107(1): 40-45.

50. Stricker SH, Feber A, Engström PG, Carén H, Kurian $\mathrm{KM}$, et al. (2013) Widespread resetting of DNA methylation in glioblastoma-initiating cells suppresses malignant cellular behavior in a lineagedependent manner. Genes Dev 27(6): 654-669.

51. Kim J, Hoffman JP, Alpaugh RK, Rhim AD, Reichert M, et al. (2013) An iPSC line from human pancreatic ductal adenocarcinoma undergoes early to invasive stages of pancreatic cancer progression. Cell Rep 3(6): 2088-2099.

52. Kim J, Zaret KS (2015) Reprogramming of human cancer cells to pluripotency for models of cancer progression. Embo J 34(6): 739-747.

53. Williams AS, Shawwa A, Merrimen JD, Haché K (2016) Expression of OCT4 and SALL4 in Diffuse Large B-cell Lymphoma: An Analysis of 145 Consecutive Cases and Testicular Lymphomas. Am J Surg Pathol 40: 950957.

54. Saxe JP, Tomilin A, Schöler HR, Plath K, Huang J (2009) Post-translational regulation of Oct4 transcriptional activity. PLoS ONE 4(2): e4467.

55. Vilchez D, Boyer L, Morantte I, Lutz M, Merkwirth C, et al. (2012) Increased proteasome activity in human embryonic stem cells is regulated by PSMD11. Nature 489: 304-308.

56. Buckley SM, Aranda-Orgilles B, Strikoudis A, Apostolou E, Loizou E, et al. (2012) Regulation of pluripotency and cellular reprogramming by the ubiquitin-proteasome system. Cell Stem Cell 11(6): 783-798.

57. Hoof DV, Muñoz J, Braam SR, Pinkse MW, Linding R, et al. (2009) Phosphorylation dynamics during early differentiation of human embryonic stem cells. Cell Stem Cell 5: 214-226.

58. Ngo VN, Davis RE, Lamy L, Yu X, Zhao H, et al. (2006) A loss-of-function RNA interference screen for molecular targets in cancer. Nature 441: 106-110.

59. Segal E, Friedman N, Kaminski N, Regev A, Koller D (2005) from signatures to models: understanding cancer using microarrays. Nat Genet 37: S38-45. 


\section{Journal of Embryology \& Stem Cell Research}

60. Wei CL, Miura T, Robson P, Lim SK, Xu XQ, et al. (2005) Transcriptomic profiling of human and murine ESCs identifies divergent paths required to maintain the stem cell state. Stem Cells 23(2): 166185.

61. Boyer LA, Lee TI, Cole MF, Johnstone SE, Levine SS, et al. (2005) Core transcriptional regulatory circuitry in human embryonic stem cells. Cell 122: 947-956.

62. Kim J, Woo AJ, Chu J, Snow JW, Fujiwara Y, et al. (2010) A Myc network accounts for similarities between embryonic stem and cancer cell transcription programs. Cell 143: 313-324.

63. Ramsay RG, Gonda TJ (2008) MYB function in normal and cancer cells. Nat Rev Cancer 8(7): 523-534.

64. Whitfield JR, Soucek L (2012) Tumor microenvironment: Becoming sick of Myc. Cell Mol Life Sci 69: 931-934.

65. Oronsky B, Reid TR, Oronsky A, Caroen S, Carter CA, et al. (2018) Brief report: RRx-001 is a c-Myc inhibitor that targets cancer stem cells. Oncotarget 9: 23439-23442.

66. Lapidot T, Sirard C, Vormoor J, Murdoch B, Hoang T, et al. (1994) A cell initiating human acute myeloid leukaemia after transplantation into SCID mice. Nature 367: 645-648.

67. Al-Hajj M, Wicha MS, Benito-Hernandez A, Morrison SJ, Clarke MF (2003) Prospective identification of tumorigenic breast cancer cells. Proc Natl Acad Sci USA 100(7): 3983-3988.

68. Kim CF, Jackson EL, Woolfenden AE, Lawrence S, Babar I, et al. (2005) Identification of bronchioalveolar stem cells in normal lung and lung cancer. Cell 121(6): 823-835.

69. Burns JS, Abdallah BM, Guldberg P, Rygaard J, Schrøder HD, et al. (2005) Tumorigenic heterogeneity in cancer stem cells evolved from long-term cultures of telomerase-immortalized human mesenchymal stem cells. Cancer Res 65(8): 3126-3135.

70. Collins AT, Berry PA, Hyde C, Stower MJ, Maitland NJ (2005) Prospective identification of tumorigenic prostate cancer stem cells. Cancer Res 65: 1094610951.

71. Piccirillo SG, Reynolds BA, Zanetti N, Lamorte G, Binda E, et al. (2006) Bone morphogenetic proteins inhibit the tumorigenic potential of human brain tumour-initiating cells. Nature 444: 761-445.

72. O'Brien CA, Pollett A, Gallinger S, et al. (2007) A human colon cancer cell capable of initiating tumour growth in immunodeficient mice. Nature 445: 106110.

73. Liu N, Li S, Wu N, Cho KS (2017) Acetylation and deacetylation in cancer stem-like cells. Oncotarget 8: 89315-89325.

74. Chen X, Sun K, Jiao S, Cai N, Zhao X, et al. (2014) High levels of SIRT1 expression enhance tumorigenesis and associate with a poor prognosis of colorectal carcinoma patients. Sci Rep 4: 7481-7491.

75. Voutsadikis I (2018) The pluripotency network in colorectal cancer pathogenesis and prognosis: An update Biomarker Med 12(6): 653-665.

76. Cao L, Li C, Shen S, Yan Y, Ji W, et al. (2013) OCT4 increases BIRC5 and CCND1 expression and promotes cancer progression in hepatocellular carcinoma. BMC Cancer 13: 82-89.

77. Zhou X, Huang GR, Hu P (2011) Over-expression of Oct4 in human esophageal squamous cell carcinoma. Mol Cells 32(1): 39-45.

78. Lin J, Zhang L, Huang $H$, Huang $Y$, Huang L, et al. (2015) MiR-26b/KPNA2 axis inhibits epithelial ovarian carcinoma proliferation and metastasis through downregulating OCT4. Oncotarget 6(27): 23793-23806.

79. Piva M, Domenici G, Iriondo O, Rábano M, Simöes BM, et al. (2014) Sox2 promotes tamoxifen resistance in breast cancer cells. EMBO Mol Med 6(1): 66-79.

80. Bass AJ, Watanabe $\mathrm{H}$, Mermel $\mathrm{CH}, \mathrm{Yu} \mathrm{S}$, Perner S, et al. (2009) SOX2 is an amplified lineage-survival oncogene in lung and esophageal squamous cell carcinomas. Nat Genet 41: 1238-1242.

81. Sodja E, Rijavec M, Koren A, Sadikov A, KoroŠec P, et al. (2016) The prognostic value of whole blood SOX2, NANOG and OCT4 mRNA expression in advanced small-cell lung cancer. Radiol Oncol 50(2): 188-196.

82. Yamawaki K, Ishiguro T, Mori Y, Yoshihara K, Suda K, et al. (2017) Sox2-dependent inhibition of p21 is associated with poor prognosis of endometrial cancer. Cancer Sci 108(4): 632-640. 


\section{Journal of Embryology \& Stem Cell Research}

83. Schneider CA, Rasband WS, Eliceiri KW (2012) NIH Image to Image J: 25 years of image analysis. Nature Methods 9(7): 671-675.

84. Kim D, Geo Pertea, Cole Trapnell, Harold Pimentel, Ryan Kelley, et al. (2013) Top Hat2: accurate alignment of transcriptomes in the presence of insertions, deletions and gene fusions. Genome Biol 14(4): R36.

85. Trapnell C, David G Hendrickson, Martin Sauvageau, Loyal Goff, John L Rinn, et al. (2013) Differential analysis of gene regulation at transcript resolution with RNA-seq. Nature biotechnology 31: 46-53.

86. Theodorou E, Dalembert G, Heffelfinger C, White E, Weissman S, et al. (2009) A high throughput embryonic stem cell screen identifies Oct-2 as a bifunctional regulator of neuronal differentiation. Genes Dev 23(5): 575-588.

87. Buckley SM, Aranda-Orgilles B, Strikoudis A, Apostolou E, Loizou E, et al. (2012) Regulation of pluripotency and cellular reprogramming by the ubiquitin-proteasome system. Cell Stem Cell 11(6): 783-798.

88. Thome M, Charton JE, Pelzer C, Hailfinger S (2010) Antigen receptor signaling to NF-kappaB via CARMA1, BCL10, and MALT1. Cold Spring Harb Perspect Biol 2(9): a003004. 